\title{
Fuzzy Calibration of Magnetometer in Presence of Surgical Microscope
}

\author{
Chanikarn Benjavitvilai and Cameron N. Riviere \\ The Robotics Institute, Carnegie Mellon University, Pittsburgh, Pa., USA
}

\begin{abstract}
A method is presented for data-driven calibration of the magnetometer in an active handheld microsurgical instrument in the presence of a surgical microscope. The instrument is designed to provide tremor compensation during vitreoretinal microsurgery. An adaptive-network-based fuzzy inference system is used to generate a calibration based on data points collected at random locations throughout the workspace. Inputs to the model include the tilt angle and the twist angle about the long axis of the instrument, obtained from accelerometer readings, and the azimuth of the apparent magnetic north, obtained from the magnetometer readings. The model outputs the true azimuth angle of the instrument. Results from preliminary testing of the method are presented.
\end{abstract}

\section{INTRODUCTION}

$\mathrm{S}$ UPPRESSION of physiological tremor for enhanced accuracy during microsurgery has been the subject of several research efforts in recent years [1-3]. One such effort has been the development of Micron, a fully handheld active micromanipulator [4]. This instrument is designed to sense its own rigid-body motion, distinguish in real time between desired and erroneous components of motion, and then deflect its own tip in a manner equal but opposite to the undesired components, thus compensating them.

Motion sensing in Micron is performed using an allaccelerometer inertial measurement unit [5]. A displacement signal can be obtained from this unit by integration of the accelerometer signals, but this estimate is corrupted by integration drift. In inertial motion sensing applications, a Kalman filter is often used to combine the integrated displacement signal with a separate drift-free estimate of position or orientation (e.g., GPS) in order to counteract the drift [6]. The accelerometers onboard the instrument provide a measurement of the direction of gravity that can be used to counteract drift in the tilt angle, as well as in the twist angle (representing rotation about the long axis of the instrument). Since the gravity vector provides no information about the azimuth, a separate source of this information is needed. A triaxial magnetometer has therefore been built into the instrument [7].

Calibration of strapdown magnetometers typically involves estimating the bias, scale factor, and misalignment of each sensor, as well as accounting for any errors due to other sources of magnetic fields located on board the vehicle or device [8]. Unwanted magnetic fields caused by items external to the device are generally ignored [8]. Application of such a technique to the present application is problematic, however. Instruments for vitreoretinal microsurgery are used in close proximity to the surgical microscope. Much of the microscope is made of steel, which creates distortion of the magnetic field sensed by the magnetometers. In addition, the the viewing light inside the microscope is turned on during surgery, creating an additional magnetic field. Calibrating for this effect is a problem because the instrument, as presently designed, has no information about the location of the microscope. Therefore the presence of the microscope represents an unknown source of magnetic field that distorts the measurement of the direction of magnetic north.

The unknown location of the microscope limits the range of techniques available for development of a useable calibration for the estimation of azimuth in this application. One possibility that remains is a data-driven approach. Data can be sampled from many positions in the workspace, and then online interpolation between these results can be used to determine the azimuth at each point during operation. Implementation of this approach is facilitated by the fact that during vitreoretinal microsurgery the motion of the instrument is constrained by its insertion through the sclerotomy, or hole in the white of the eye, that enables the tool to reach the retina. This reduces the dimensionality of the space that the calibration data set must cover. Not only can such a calibration method account for the presence of the microscope, but in fact it obviates calibration of the scale factor of the individual sensors. Rather than calibrating sensors and then generating a mapping from calibrated magnetic field measurements to azimuth, it is just as straightforward to generate instead a mapping directly from uncalibrated magnetometer readings to azimuth.

The discrete nature of the data points in an interpolation data set makes the application ideally suited to fuzzy computation [9]. This paper presents an application of an adaptive-network-based fuzzy inference system [10] to calibration of the magnetometer in Micron in the presence of the surgical microscope.

Funding provided by National Science Foundation (grant no. EEC9731748) and National Institutes of Health (grant no. 1 R01 EB000526). 


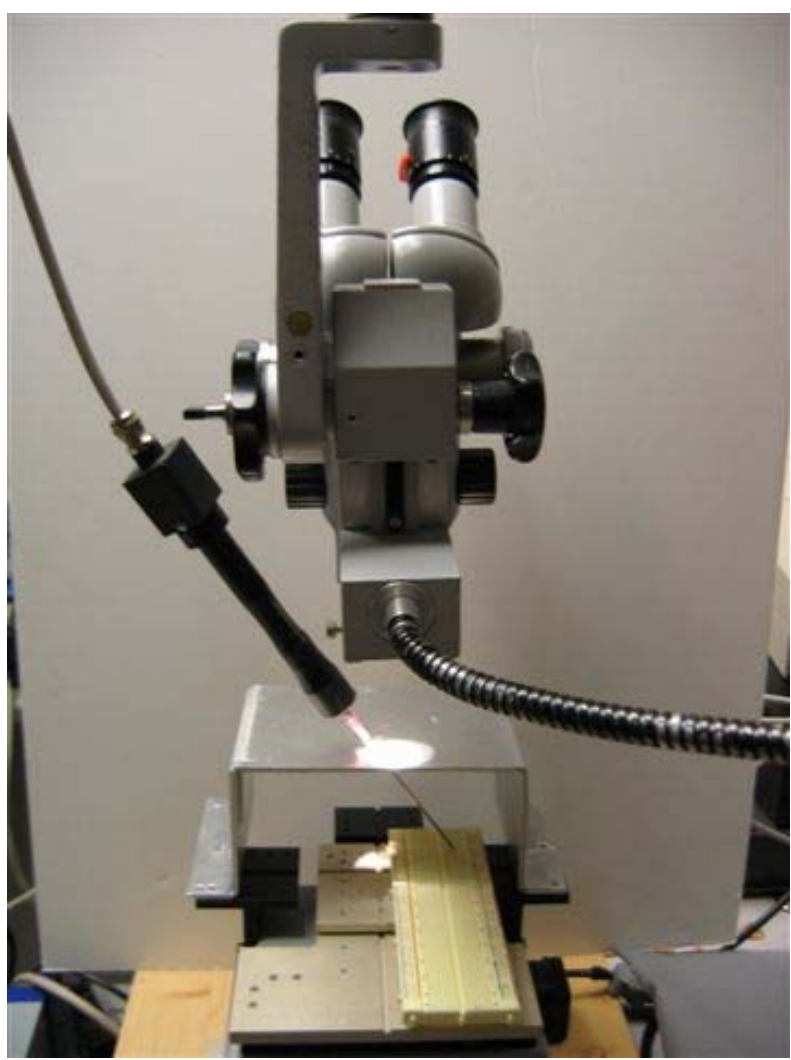

Fig. 1. The experimental setup. The instrument is inserted through a small hole in a plate in simulation of a sclerotomy. The microscope is set to maximum illumination, and focused on a spot roughly $1 \mathrm{~cm}$ from the sclerotomy, where the pupil would typically be. A standard 20 -gauge needle is affixed to the tip of the instrument. The needle is inserted into a length of steel hypodermic tubing in order to form a longer tool shaft. Different testing axes (tilt $\&$ azimuth angles) were selected by inserting the shaft in different holes in the breadboard seen below the plate.

\section{METHODS}

\section{A. Data Acquisition}

The experimental setup is illustrated in Fig. 1. The instrument was inserted through a hole in an aluminum plate in simulation of a sclerotomy. The hole diameter was roughly $1 \mathrm{~mm}$. A 20-gauge needle was affixed to the tip of the instrument. On the underside of the plate, a length of stainless steel hypodermic tubing was affixed over the needle to provide a longer tool shaft. Below the aluminum plate was affixed a standard circuit-prototyping breadboard, at a vertical distance of $69.1 \mathrm{~mm}$. The tip of the hypodermic tubing was tapered so that it would enter into the holes in the breadboard and not slip out easily. Tilt and azimuth angle for a trial were therefore chosen by inserting the shaft in a given hole in the breadboard.

The microscope was brought to a realistic operating distance $(10 \mathrm{~cm})$ from the plane of the sclerotomy, and was focused on a point $1 \mathrm{~cm}$ from the sclerotomy as an approximation of the typical horizontal distance between pupil and sclerotomy. The viewing light was turned to its maximum intensity.

The space of reachable orientations is irregularly shaped because of the length of the instrument and the irregular shape of the microscope. For data collection, 27 points were chosen at random, roughly covering the reachable workspace. The orientations tested ranged in azimuth from $56^{\circ}$ to $127^{\circ}$, and in tilt from $22^{\circ}$ to $38^{\circ}$. At each orientation tested, the signals from three magnetometers and six accelerometers were recorded as the instrument was twisted a full $360^{\circ}$ about its long axis. The azimuth value for each orientation was measured from the physical apparatus using a caliper.

\section{B. ANFIS Model}

The ANFIS modeling toolbox in Matlab (The MathWorks, Natick, Mass.) was used to generate the model. There were five inputs to the model: the twist angle and tilt angle (obtained from accelerometer readings), and the three orthogonal magnetometer readings. The output of the model is the azimuth angle.

\section{RESULTS}

The ANFIS model performance on the training set is depicted in Fig. 2. The root mean square error for the training set is $1.27^{\circ}$. Fig. 3 displays the performance on the testing set. The root mean square error for the testing set is $5.07^{\circ}$.

\section{DISCUSSION}

The results demonstrate the feasibility of data-driven calibration of the magnetometers in Micron using a fuzzy inference system such as ANFIS. Experimentation in our laboratory has shown that the magnitude of the distortion in the magnetometer signals due to the microscope, particularly when the viewing light is on, can sometimes be more than half the magnitude of the sensed earth magnetic vector itself. The rmse value of $5.07^{\circ}$ is far lower than would be obtained

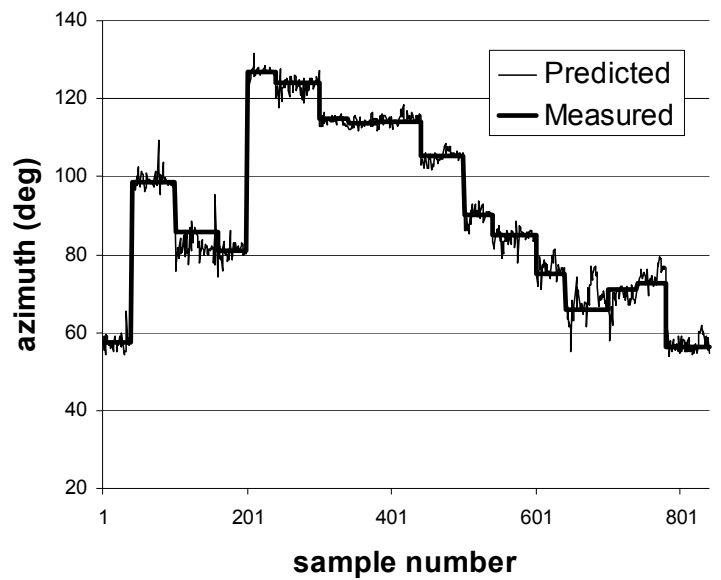

Fig. 2. Results for the training data set. The data set contains 840 points collected along 18 axes. 


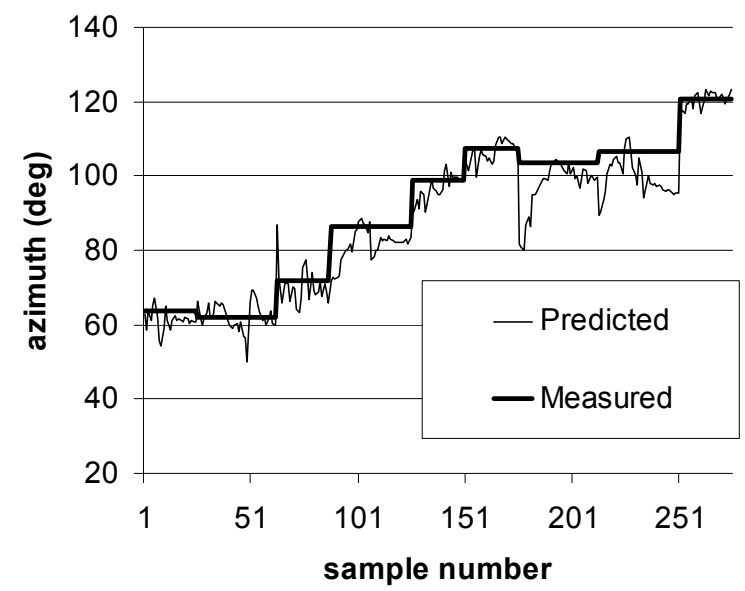

Fig. 3. Results for the testing data set. The data set contains 277 points collected along 9 axes.

using a calibration that did not take the microscope into account. It is hoped that further improvements in performance can be obtained by collecting a larger data set that incorporates more different tilt and azimuth values in the model.

\section{REFERENCES}

[1] I. W. Hunter, T. D. Doukoglou, S. R. Lafontaine, P. G. Charette, L. A. Jones, M. A. Sagar, G. D. Mallinson, and P. J. Hunter, "A teleoperated microsurgical robot and associated virtual environment for eye surgery," Presence, vol. 2, pp. 265-280, 1993.

[2] H. Das, H. Zak, J. Johnson, J. Crouch, and D. Frambach, "Evaluation of a telerobotic system to assist surgeons in microsurgery," Comput. Aided Surg., vol. 4, pp. 15-25, 1999.

[3] R. Taylor, P. Jensen, L. Whitcomb, A. Barnes, R. Kumar, D. Stoianovici, P. Gupta, Z. Wang, E. de Juan, Jr., and L. Kavoussi, "A steady-hand robotic system for microsurgical augmentation," Int. J. Robot. Res., vol. 18, pp. 1201-1210, 1999.

[4] C. N. Riviere, W. T. Ang, and P. K. Khosla, "Toward active tremor canceling in handheld microsurgical instruments," IEEE Trans. Rob. Autom., vol. 19, pp. 793-800, 2003.

[5] W. T. Ang, P. K. Khosla, and C. N. Riviere, "Design of allaccelerometer inertial measurement unit for tremor sensing in handheld microsurgical instrument," Proc. IEEE Intl. Conf. Robot. Autom., Taipei, Taiwan, Sept. 14-19, 2003, pp. 1781-1786.

[6] A. H. Mohamed, K. P. Schwarz, "Adaptive Kalman filtering for INS/GPS,” J. Geodesy, vol. 73, no. 4, pp. 193-203, May 1999.

[7] W. T. Ang, P. K. Khosla, and C. N. Riviere, "Kalman filtering for real-time orientation tracking of handheld microsurgical instrument," Proc. IEEE/RSJ Intl. Conf. Intell. Robots Syst. (IROS), Sept. 28-Oct. 2, 2004, vol. 3, pp. 2574-2580.

[8] D. Gebre-Egziabher, G. H. Elkaim, J. D. Powell, and B. W. Parkinson, "A non-linear, two-step estimation algorithm for calibrating solid-state strapdown magnetometers," Proc. $8^{\text {th }}$ St. Petersburg Intl. Conf. Integrated Navigation Systems, St. Petersburg, Russia; May 28-30, 2001. pp. 290-297.

[9] L. C. Westphal, "Fuzzy inference systems and artificial neural networks for continuous piecewise multilinear interpolation," Electron. Lett., vol. 34, no. 18, pp. 1764-1766, 1998.

[10] J.-S. R. Jang, "ANFIS: adaptive-network-based fuzzy inference system," IEEE Trans. Syst. Man Cybern. vol. 23, no. 3., pp. 665-685. 